\title{
Sustentabilidad: Liderar Organizaciones Migrantes en el Uruguay- Aspectos Asociados al Alto Desempeño Empresarial
}

\author{
Alberto González ${ }^{1^{*}}$, Natalia Mandirola ${ }^{1}$, John Miles ${ }^{1}$
}

Resumen: Las empresas son organizaciones cuya actividad principal son los negocios y se suele confundir ambos aspectos: el negocio y la organización. La experiencia muestra que las empresas que permaecen en el tiempo se caracterizan por gestionar y evolucionar la relación organizaciónnegocio en principalmente tres modalidades: mejorando su modelo de negocio, integrando nuevos negocios y migrando a otros negocios.

Es cada vez más necesario para la supervivencia de una organización, el fortalecer dicha competencia específica de "migrar": capacidad de desvincularse de un (modelo de) negocio y asumir otro (modelo de) negocio.

Esta realidad impacta diferente a cada stakeholder. Los propietarios pueden migrar con mayor facilidad si deciden retirar su capital, vender el negocio, etc. El costo para los trabajadores es superior, afecta su fuente de trabajo y las condiciones de vida familiar, hasta su inclusión o exclusión social. Para la comunidad, la caída o reconversión de una organización puede implicar un costo colectivo enorme.

El pensamiento económico y administrativo ha tendido a focalizar el problema en la sustentabilidad del negocio, pero desde la mirada ética y pasando por la Doctrina Social de la Iglesia, la sustentabilidad de la organización resulta más importante que la sustentabilidad del negocio.

Se entrevistaron 55 empresas uruguayas que han perdurado más de 30 años, soportando radicales cambios del entorno, manteniendo posiciones de primera línea en sus áreas de acción. Un análisis cuantitativo de sus respuestas ilustra los factores de alto desempeño de su gestión, y un análisis cualitativo identifica características asociadas a su sustentabilidad organizacional, las cuales se presentan mediante el concepto de organizaciones migrantes.

Los resultados muestran que además de las competencias de gestión, importan los valores y las actitudes de la dirección, confirmando la relevancia que tiene para las escuelas de negocios la formación de líderes conscientes de sus decisiones.

Palabras clave: Sustentabilidad; Ética empresarial; Migración organizacional; Modelo de negocio

Abstract: Companies are organizations whose main activities are businesses and both aspects are often confused: the business and the organization. Evidence shows that long-lasting companies are characterized by their capability of managing and evolving the relationship between the organization and its business in three main ways: improving its business model, integrating new businesses and migrating to other businesses.

It is increasingly necessary for the survival of an organization, to strengthen that specific competence of "migration": the ability to opt out of a business (model) and incorporate another business (model).

This fact implies different impacts among each stakeholder. Owners can migrate more easily if they decide to withdraw their money, sell the business, etc. The cost is higher for workers, affecting their jobs incomes, family life conditions, and even its inclusion or social exclusion. For the community, the collapse or conversion of an organization can cause a huge collective cost.

Economic and management thinking have tended to focus the problem on business sustainability, but considering and ethics and The Catholic's Social Doctrine approaches, the sustainability of the organization is more important than business sustainability.

55 Uruguayan companies that have lasted over 30 years were interviewed, supporting radical changes of their environment, keeping front-line positions in their areas of action. A quantitative analysis of responses illustrated the high performance factors of their management, and a qualitative analysis identified characteristics associated to its organizational sustainability, which are presented through the concept of migrant organizations.

Results showed that in addition to management skills, values and attitudes of leaders where also determinant, confirming the relevance for Business Schools to train leaders aware of their decisions.

Keywords: Sustainability; Business Ethics; Organizational Migration; Business Model

$21^{\text {st }}$ International Association of Jesuit Business Schools (IAJBS)

$18^{\text {th }}$ Colleagues in Jesuit Business Education (CJBE)

(1) Departamento Ciencias de la Administración, Facultad de Ciencias Empresariales, Universidad Católica del Uruguay

*Corresponding author: agonzale@ucu.edu.uy

ISSN: 0718-2724. (http://jotmi.org)

Journal of Technology Management \& Innovation ( ) Universidad Alberto Hurtado, Facultad de Economía y Negocios.

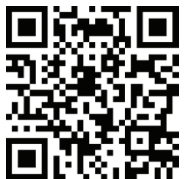




\section{Introducción}

La sustentabilidad de una organización empresarial en el largo plazo no se confunde con la sustentabilidad del negocio ni de la inversión. La organización puede cambiar de negocio, forma específica en la que genera valor para el cliente en un determinado momento, de la misma manera que el inversor puede cambiar de organización. En el primer caso, la organización seguirá cumpliendo su papel de sustento a las familias de sus trabajadores y su aporte a la sociedad mediante una mudanza de negocio, mientras que en el inversor, orientado a la rentabilidad de la acción a corto plazo salva su capital en forma independiente con lo que suceda en la organización (Barton y Wiseman, 2014). El mecanismo de la propiedad por acciones y la cotización en bolsa fortalece justamente esta posibilidad: salvar a propietarios aunque se perjudiquen trabajadores y otros actores. Cuando se observa desde el conjunto de los grupos de interés sostener la organización es una responsabilidad ética.

En entornos aceleradamente cambiantes, donde las ventajas competitivas que disponga cualquier negocio pueden desaparecer rápidamente (Gunther, 2013), abordar la permanencia en el tiempo de las organizaciones -más allá del negocio- es clave también por el costo que significa la desaparición de una empresa (Davis, 2014a): pérdidas de activos tangibles, ineficiente vía de innovación, etc.

Cuando la desaparición de empresas tiene un alto impacto (por la historia de la empresa, por la presión de los trabajadores, por el costo económico/social, etc.) no es raro que el poder político intervenga para mantener la fuente de trabajo. Sin embargo, la experiencia uruguaya muestra que a veces la intervención o el apoyo del estado puede ser ingenuo en el sentido que principalmente se abra a cambio en la forma de propiedad, por ejemplo de propiedad individual a propiedad cooperativa, pero que ignore las necesarias competencias de gestión que la organización como empresa debe asumir para ser viable.

El análisis que se presenta en este trabajo, focalizado en la sustentabilidad organizacional indaga en cuáles son prioridades de gestión de los directivos, las características de las prácticas de dirección asociadas a la sustentabilidad organizacional e identifica una tipología de migración de negocios en empresas sustentables. Es parte de una investigación mayor sobre prácticas de dirección en empresas uruguayas de alto desempeño.

\section{Marco Conceptual}

\subsection{Sustentabilidad Organizacional}

La sustentabilidad de las organizaciones a largo plazo es una problemática que dispone de abundante y plural literatura. Wikström (2010) muestra enfoques más allá de la sustentabilidad ambiental, incorporando como Thomas y Lamm (2012) la legitimidad social como otra dimensión. Este trabajo apunta a un concepto cercano a Normas ISO 9004 (2009) que implica la permanencia de la organización en el tiempo con capacidad de satisfacer en forma razonable al conjunto de los grupos de interés (stakeholders) en períodos que superan un ciclo de negocios o de innovación. Davis (2014a) destaca este punto para identificar y evidenciar competencias que son independientes de un viento de cola favorable o de un posicionamiento circunstancial (Smit et al., 2005).

Poder permanecer más allá del ciclo de negocio lleva a apoyarse en la teoría de la empresa basada en recursos (resource-based theory) (Wernerfelt, 1984; Barney, 1991) y en particular de capacidades dinámicas (Teece y Pisano, 1994) por cuanto éstas serían las que permiten superar los ciclos.

\subsection{Empresas y organizaciones}

El foco en la organización y no en la empresa implica poner énfasis en el grupo humano que la conforma y el que la rodea (comunidad) y no tanto en el negocio en sí mismo, que se transforma en la base instrumental para la subsistencia de la organización y no hacer del negocio, la finalidad de la organización. Este énfasis en la organización se aplica como herramienta de estudio, pero no debe interpretarse como una propuesta de gestión llevada al extremo, en tanto que (a) muchas organizaciones no son finalidades en sí mismas, como por ejemplo, las que prestan servicios de salud, (b) el negocio asegura la sustentabilidad de la organización en cuanto empresa.

El concepto de modelo de negocio está teniendo una importancia creciente como herramienta conceptual -aunque con gran amplitud de enfoques (Shafer et al., 2005)- y también práctico, como muestra el uso del "Canvas" de Osterwalder y Pigneur (2010). Su definición de modelo de negocio - "describe el mecanismo por el cual una organización crea, entrega y capta valor”- es útil para analizar la dialéctica entre organización y empresa. La empresa es una organización provista de un (o varios) modelo(s) de negocio que evolucionan en el tiempo. Una manera de analizar la sustentabilidad a largo plazo es relacionarla con la forma en la cual la organización se articula con el negocio y con el modelo de negocio. Toda empresa reposa en la gestión de por lo menos un negocio, pero la permanencia en el tiempo muestra que esa relación es altamente inestable: desde cambio de modelo de negocio, hasta la migración hacia otros negocios. En realidad la sustentabilidad depende de la capacidad de la empresa de modificar su relación con el negocio procurando incluso la innovación en el modelo, (Carayannis et al., 2014). Si la empresa es incapaz de modificar su relación, no subsistirá (Brynjolfsson y McAfee, 2014). $\mathrm{Al}$ enfatizar la diferencia entre organización y empresa, se introduce el concepto de organización migrante, como aquella empresa que es capaz de cambiar tanto de modelo de negocio, como, en situación más extrema del negocio mismo. Esta explicación no pretende ser exhaustiva como indican Smit et al. (2005) y el impacto del modelo depende del ciclo de negocio en particular y del entorno industrial como exploran en un meta-análisis Rosenbusch et al. (2007). Además las dinámicas pueden cambiar bastante entre una multinacional global y una empresa nacional que son las que constituyen la mayoría de este estudio. 


\subsection{Organizaciones de alto desempeño}

El alto desempeño es una característica de la sustentabilidad y la norma ISO 9004/2009 (ISO, 2009) comparte una similar definición de organización de alto desempeño afirmando que se trata de una gestión para el éxito sostenido de una organización en el tiempo satisfaciendo al conjunto de los grupos de interés. La investigación de Algorta et al. (2012) muestra para el caso uruguayo, que empresas que trabajan con este enfoque logran resultados sobresalientes.

En un mundo altamente dinámico, la permanencia en el tiempo está asociada a la capacidad migratoria como competencia dinámica (Teece, 2007 y 2009) tanto para adaptarse al entorno como para crear uno distinto. La capacidad de cambiar el modelo de negocio o ir más allá, migrando de negocio está asociada a las modalidades y estilos de gestión, pero también a los valores de la dirección de la empresa, aunque como indica Gerstner (2014) los mismos son operativos cuando se traducen en acciones y reconocimiento al personal comprometido con ellos.

En la línea de la sustentabilidad de empresas exitosas, se ha elegido el marco conceptual de André de Waal (2012) que identifica 5 factores que resumen 35 características de las prácticas de la alta dirección en organizaciones de alto desempeño:

1) Alta calidad del equipo directivo

2) Apertura y orientación a la acción

3) Compromiso a largo plazo

4) Mejora continua y renovación

5) Alta calidad de los empleados

Esta investigación utiliza el modelo de de Waal (2012) para construir una grilla que permita relevar e identificar características de las prácticas de la dirección en las organizaciones. Conceptualmente, es relevante indagar si la sustentabilidad tiene características de gestión comunes entre las diferentes empresas que puedan ir más allá de los fuertes cambios del entorno que afectan la permanencia.

\section{Objetivo y metodología}

\subsection{Objetivo}

El objetivo del trabajo es mostrar cuáles son características de las prácticas de dirección que muestran las empresas privadas uruguayas que permanecen competitivamente en el mercado desde hace varios ciclos de negocios mostrando aspectos relevantes de satisfacción del conjunto de los grupo de interés (stakeholders) en clave de sustentabilidad organizacional y caracterizar las formas de migración de negocios.

\subsection{Muestra de estudio}

Los resultados aquí presentados están basados en entrevistas realizadas a 56 a altos directivos de 55 empresas seleccionadas por una muestra intencional, según estos criterios:

1) Empresas privadas nacionales grandes o medianas de diferentes ramas de actividad situadas en contexto competitivo, presentes en más de un ciclo de negocios en su área, que ocupan lugares preferenciales en su sector y que no hayan generado problemas importantes con alguna de las partes interesadas.

2) Empresas de origen nacional, adquiridas por multinacionales, cuya permanencia en el país se muestra en ambiente competitivo y que mantienen rasgos de una fuerte cultura de gestión nacional.

Importa destacar que quedaron fuera de la muestra empresas de alto desempeño que fueran filiales de multinacionales.

Perfil de los entrevistados: directivos ejecutivos de la organización, propietarios y/o fundadores o directivos de alta gerencia (gerentes generales o equivalentes), es decir, quienes tienen un impacto fuerte en la gestión y no son meros ejecutores, con permanencia asociada a la trayectoria exitosa de la organización, contando al menos, 5 años de desempeño de dichas tareas directivas.

Se relevaron 55 empresas, 38 nacionales, 11 multilatinas o con alguna presencia internacional y 6 multinacionales (5 compraron empresas nacionales). Según los criterios nacionales, 24 Pymes y 31 grandes.

\subsection{Campo}

Las entrevistas duraban de 50 a 90 minutos y comprendía una estructura mixta (cuanti y cuali) con 39 preguntas de base, ordenadas en 4 secciones:

1) Ordenamiento de los factores y características del modelo de de Waal en cuanto su relevancia para la organización y grado de avance. La indagación sobre ejemplos de prácticas asociadas a los factores y sus características permite controlar la interpretación de la pregunta y el sentido dado por el entrevistado.

2) Preguntas sobre otros factores o características de las prácticas de gestión que pudieran estar ausentes del modelo anterior.

3) Indagación de los hitos -momentos en los cuales la empresa vivió un "antes y después"- asociados, sea a una situación que ponía en riesgo la continuidad de la misma, sea a una situación que implicaba un cambio importante. El relato de los hitos permite evidenciar actitudes, comportamientos, valores en la toma de decisiones significativos para la permanencia y que a veces quedan ocultos en la cotidianidad de la gestión.

4) Información general sobre el directivo, la empresa y sus resultados en los últimos tres años.

\subsection{Enfoque analítico}

La información obtenida responde a un diseño mixto que además de análisis estadísticos y cualitativos separados, permite la articulación de los contenidos (Brady y Collier, 2010).

En consecuencia estamos ante un estudio exploratorio y descriptivo, cuyos resultados permiten generar una interpretación y un conjunto de hipótesis aptos para futuros desarrollos. La descripción de las características de dirección observadas en estas empresas no implica 
que sean exclusivas de las mismas, pero razonablemente responden a ciertos perfiles empresariales que aparecen asociados a la permanencia en el largo plazo y el éxito empresarial.

El estudio es consciente del sesgo implícito que puede surgir de la autoevaluación de su propio comportamiento realiza por los directivos. Estudios locales anteriores indican que si bien los ejecutivos no están exentos de sesgos en sus perspectivas, pueden ser fuentes confiables sobre muchas prácticas y realidades de sus organizaciones (Miles, 2011). El uso de escalas de preferencia entre factores todos ellos positivos permite reducir este efecto y la jerarquía de prácticas encontrada puede ajustar razonablemente a la realidad. También se obtuvieron comentarios autocríticos fuertes en no pocos casos, tanto de la situación organizacional como del propio desempeño.

\subsection{Entorno económico como contexto del análisis}

El promedio de antigüedad de las empresas entrevistadas es de 45 años con un máximo de 121 años. Seguramente el período reciente ha influenciado las actuales culturas organizativas que son las que se pueden indagar. En los últimos 40 años, existieron acontecimientos y cambios nacionales muy radicales y de alto impacto.

Bien que el Uruguay en el siglo XX fue predominantemente una democracia, en el año 1973 sufrió un golpe de estado que instaló una dictadura cívico-militar hasta 1985. La dictadura fue precedida por un largo período de bajo crecimiento económico. En 1982 hay una brutal caída del PIB de -9,3\%. A partir de 1985 el PIB ofrece niveles positivos de crecimiento, pero muy oscilante de año en año, y luego de tres años de retroceso se produce otra brutal caída en el año 2002 del $-7,1 \%$, caída del $22 \%$ del salario real (Durán et al., 2012) y con niveles de desempleo del $17 \%$ y de pobreza de casi el $40 \%$, cuando esta última, en años anteriores era del orden del 17\%. A partir de 2003 comienza un período de alto crecimiento único en el país por su intensidad y duración (Vázquez, 2015), logrando mínimos históricos en los indicadores de desempleo y pobreza. Las crisis de 1982-83 y de 1999-2002 produjeron el cierre de numerosas empresa y en ambas estallaron la desocupación y produjeron amplia desintegración del tejido social.

Las políticas económicas de los últimos 40 años también oscilaron fuertemente, desde prácticas básicamente liberales a orientaciones más reguladoras pero en general con tendencia a la apertura externa y recientemente, con creciente peso de organizaciones sociales y sindicatos.

En síntesis, si bien los últimos 12 años han sido muy favorables, las empresas visitadas y la mayoría de los directivos entrevistados han vivido entornos muy cambiantes y momentos terriblemente críticos y su supervivencia implica capacidad de sustentabilidad (Man y Lau, 2000) y en este contexto hay que interpretar los relatos empresariales.

\section{Presentación de hallazgos}

Los resultados que se presentan en este trabajo resultan de una combinación de los aspectos cuantitativos y los cualitativos de la investigación, apoyado en ejemplos con los cuales se les pedía ilustrar las opiniones de los directivos para asegurar la interpretación de los relatos.

\subsection{Características de las prácticas de dirección destacadas}

Los directivos entrevistados debían ordenar los factores del alto desempeño según la importancia relativa para su organización. Llevado a una escala de 0 a 10, el gráfico 1, muestra el ordenamiento de las preferencias declaradas y del avance percibido.

Gráfico 1: Relevancia asignada y avance percibido de los factores de alto desempeño

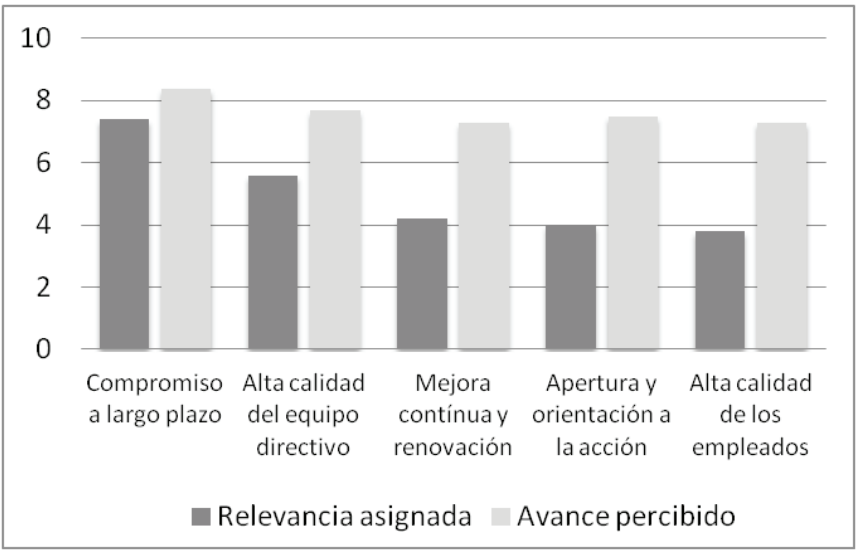

Nota: Cada directivo ordena los 5 factores según la relevancia asignada y el avance percibido. La escala con un mínimo de 0 y máximo de 10 expresa los promedios de ordenamiento. 10 indica que todos pusieron el factor como prioritario; 0 que todos lo pusieron último y 5 un nivel medio.

A su vez, para cada factor, la característica más destacada se expresa en la tabla 1.

Tabla 1: Característica más destacada para cada factor de alto desempeño

\begin{tabular}{|l|l|}
\hline FACTOR & $\begin{array}{l}\text { CARACTERTÍSTICA } \\
\text { MÁS DESTACADA }\end{array}$ \\
\hline Compromiso a largo plazo & $\begin{array}{l}\text { La organización se orienta a fidelizar } \\
\text { y a servir al cliente lo mejor posible }\end{array}$ \\
\hline Alta calidad del equipo directivo & $\begin{array}{l}\text { Los directivos son un fuerte modelo } \\
\text { de conductas ejemplares }\end{array}$ \\
\hline Mejora contínua y renovación & $\begin{array}{l}\text { La organización innova y renueva sus } \\
\text { competencias, productos, servicios y } \\
\text { procesos }\end{array}$ \\
\hline Apertura y orientación a la acción & $\begin{array}{l}\text { Los directivos promueven la apertu- } \\
\text { ra al cambio en la organización y los } \\
\text { empleados }\end{array}$ \\
\hline Alta calidad de los empleados & $\begin{array}{l}\text { La fuerza laboral es diversa y comple- } \\
\text { mentaria }\end{array}$ \\
\cline { 2 - 2 }
\end{tabular}




\subsection{Características de los directivos}

\section{Características de los directivos entrevistados}

Es significativo que al seleccionar las empresas por los criterios de alto desempeño y que no fueran (salvo excepción) multinacionales extranjeras, el perfil dominante resulte de empresas familiares, con una media ocupacional de 328 empleados. De las 55 entrevistas resultaron entrevistados, 24 fundadores, 12 descendientes de fundadores, 7 propietarios que se asociaron a la empresa existente que ya tenía un perfil familiar, dando como resultado 43 entrevistas a propietarios y 12 directivos a no propietarios. Se observa también una mayor profesionalización en los directivos más jóvenes. Como se verá más adelante, estos perfiles no buscados inicialmente son resultados de la investigación y levanta el interrogante sobre cuáles son las condiciones para la existencia y permanencia de un empresariado nacional, el perfil de las empresas nacionales destacadas y las características necesarias para afrontar la globalización.

\section{Orientación a largo plazo}

En concordancia con otras experiencias (Davis, 2014b), la permanencia organizacional aparece asociada a una explícita orientación a largo plazo, expresada en la voluntad de la dirección de permanecer y de continuar, tanto de la organización como en términos personales. En el relevamiento practicado, el Compromiso a largo plazo, aparece como el factor de alto desempeño al cual más directivos aspiran y también el mejor evaluado en su logro. Muchas veces este factor aparece asociado como compromiso de mantener los clientes en el largo plazo. Esta voluntad a su vez se vincula al sentimiento de una responsabilidad personal como dirección (en particular cuando son propietarios) con la empresa y su gente, en una confluencia entre el proyecto organizacional y el personal.

\section{Sentido de la trascendencia}

Vinculado al punto anterior, a nivel personal de los directivos, el proyecto empresarial suele asociarse a un sentido de trascendencia: crear o dejar una obra que perdure más allá de la persona, sea por la satisfacción por la obra en sí misma, sea por la continuidad o creación de un proyecto trans-generacional. Si la persona no es el fundador, el vínculo al fundador y la continuación de una tradición aparecen como relevantes bien que puedan aparecer cambios significativos en la vida de la organización cuando se producen los relevos generacionales en acuerdo con lo hallado por Hesselbein et al. (1998) en otros estudios. Cuando emerge la dificultad que la nueva generación asuman la continuidad del proyecto, aparece amenazada la sustentabilidad de la organización, pudiéndose observar la reducción de actividades y/o la posibilidad de la venta y si acontece con una multinacional.

\section{Liderazgo}

Al preguntar sobre las características de la alta calidad del equipo directivo, los directivos entrevistados enfatizan como más importantes los aspectos que dicen a la dimensión ética y menos importantes los que se refieren a aspectos de competencias técnicas del cargo. Esto también ha sido confirmado en una investigación cuantitativa en empresas uruguayas de software (Miles, 2011). Así, al proponer que ser Modelo de conductas ejemplares y Expresar confianza y seguridad son las características más importantes en la calidad de la dirección y también de las más logradas, indican perfiles claros de liderazgo. En muchas empresas, estas características aparecen ejemplificadas por relatos de fuerte y directa comunicación con el conjunto de los empleados que puede incluir desde la presencia en la operativa cotidiana, a alta visibilidad ante todos los funcionarios en momentos difíciles. Este comportamiento en situaciones de crisis, más allá de su papel para superarla, puede ser la base para renovar la confianza del personal en la dirección, en particular cuando la dirección se vuelve ejemplar: por ejemplo cuando precede con su propia austeridad lo que luego propondrá al personal. Pero importa destacar que la confianza en algunos directivos no es sinónimo de confianza en la organización.

\subsection{Características de la gestión interna}

\section{Contingencia de la gestión sustentable}

Los rasgos del ejercicio de la dirección que aseguran la sustentabilidad de las empresas responden a la lógica de la teoría de la contingencia organizacional (Mintzberg, 1983), en este caso no tanto desde un punto de vista estructural sino más bien de estilos de gestión. En este trabajo se destacan las tendencias generales pero las modalidades concretas de implementación dependen del tipo de empresa y para una misma empresa, del momento que está viviendo, afirmación que aparece frecuentemente desde los propios entrevistados, al indicar que destacan ciertas prioridades al momento de la entrevista, pero tiempos atrás hubieran destacado otras.

\section{La construcción de equipos comprometidos}

Los directivos destacan la necesidad y la capacidad de crear equipos fuertemente imbuidos con el proyecto empresarial y en general no se perciben como actores aislados. Pero este deseo tiene problemas para alcanzar a toda la organización. Cuando más alejado está el personal del vértice de la organización, la dirección tiende a percibirlo como más difícil de integrar.

Este distanciamiento está asociado a diferencias de clases: más allá de la voluntad de integración de la dirección, la condición objetiva del trabajador de nivel bajo en el organigrama suele acompañar una situación de estratificación social más baja asociada a una mirada crítica hacia los empresarios. Por tanto, el trabajador de base no genera espontáneamente una situación de compromiso personal con la organización. Quizás por esto, las características menos logradas según los directivos, son las que dicen al compromiso del conjunto del personal y la acción de la dirección sobre el personal de bajo rendimiento al tiempo se quejan del creciente esfuerzo y tiempo que lleva la gestión de personas y el eventual costo oportunidad que conlleva al disminuir la disponibilidad hacia actividades caracterizadas como más propias del negocio.

A esta situación de clase, se cruza hoy, un fortalecimiento de la brecha generacional: la generación del "milenio" se integra a la vida laboral en un contexto de prosperidad acompañado de tasas de bajo desempleo que no conocieron sus predecesores. Incluso en los niveles sociales más cercanos a la dirección, se muestra un distanciamiento a la empresa asociado a diferente valoración del trabajo y ansiedad en el cumplimiento de altas expectativas profesionales. 
En algunos casos, esto genera una suerte de dualidad y la empresa se divide en dos subculturas: un sector asociado a la permanencia y fidelidad al proyecto y un sector "emigrante" que abandonará la organización en la primera oportunidad, (no es raro que éstos pidan volver al descubrir que el trabajo en la nueva empresa es menos satisfactorio que el anterior), desencadenando diferentes comportamientos en la gestión de los recursos humanos según la categoría de los mismos. La separación se potencia a mayor tamaño de la organización.

\section{Conflictos y culturas organizacionales inclusivas}

El actor sindical ha adquirido una presencia mayor y más activa en el último decenio y el testimonio de los entrevistados suele insistir en ello. Algunas experiencias -sobre todo cuando las empresas son más pequeñas- muestran que existe una menor presión para generar sindicatos cuando las políticas laborales logran crear consenso en la organización, al tiempo que el trabajador percibe que la situación en su empresa es mejor que la situación de otras empresas. En las organizaciones mayores, el sindicato está presente, sea impulsado por actores externos o desde la propia empresa. La relación entre la organización y el sindicato adquiere nuevos perfiles y requiere cada vez más tiempo y esfuerzo por parte de la dirección que a veces se siente en aprietos para gestionar una situación de "conflicto inminente". Sin embargo, importa destacar que existen claramente experiencias en las cuales la relación empresa-sindicato coexiste de manera constructiva y ello constituye una de las bases de su sustentabilidad.

Este punto y el punto anterior (construcción de equipos) indican que las diferencias de clase y de generación plantean posiciones y demandas a las organizaciones que no pueden solucionarse por mero voluntarismo: más allá de la construcción de culturas organizacionales específicas, las empresas quedan atravesadas por culturas de dimensión social (clase y edad).

\section{Flexibilidad y autotransformación}

La vocación a persistir conlleva a la disponibilidad y habilidad al cambio. Los directivos entrevistados relatan más historias de cambios (más reactivos que proactivos) que de mejoras, bien que una vez procesados los mismos se los valore positivamente.

Si bien la indagación sobre los modelos de negocios no aparecía como objetivo inicial de la investigación, los mismos emergieron claramente de los relatos de hitos y de prácticas. Los 4 grandes tipos de gestión del cambio en los negocios observados permiten observar la gran flexibilidad y capacidad de transformación requerida para permanecer y esto desde los orígenes de los emprendimientos, al tiempo que sugieren la utilidad de herramientas que permitan gestionar más claramente ese tipo de cambio.

\section{Tecnología e innovación}

La tecnología y la innovación tecnológica no aparecen en general como un problema, sino como un recurso accesible. Los directivos tienen puesto los ojos en las mismas, bien que no sean creadores de innovaciones tecnológicas, recurren a las que están disponibles en el mercado: el perfil de las empresas entrevistadas no responde tanto a organizaciones creadoras de innovaciones globales sino a adaptadoras de las innovaciones al entorno nacional, pero conviene destacar la excepción de las empresas del área informática, donde la capacidad de innovación global está presente en los relatos.

\section{Crecimiento orgánico vs crecimiento "inorgánico"}

La mayoría de las empresas entrevistadas responden a un modelo de crecimiento orgánico: se financian con recursos propios y no parecen abiertas a la incorporación de socios capitalistas o inversores. Las mayores pueden romper con este modelo de crecimiento, especialmente cuando afrontan estrategias de internacionalización. El crecimiento orgánico puede implicar un techo de cristal, pero la experiencia muestra que muchas empresas nacionales exitosas, terminan siendo vendidas a multinacionales y a veces a la venta sigue el cierre o reducción de la filial, pues entra en lógicas globales y la filial nacional suele ser marginal en su negocio o su actividad trasladable a otro país.

\subsection{Características de la gestión externa}

\section{El impacto de las crisis}

La historia de estas empresas está marcada por la capacidad de subsistir más allá de crisis del entorno general, siendo particularmente dura la observada en el período 1999-2002 y que aún afecta duramente la memoria de empresas fuertes y sanas. La superación de la crisis parece estar asociada a competencias específicas, -más allá de la gestión ordinaria y otros aspectos, como arrancar de una posición financiera razonable- competencias de las cuales a veces la propia organización no es consciente. Estos contextos críticos pueden afectar a la dirección en forma personal más allá del obvio impacto económico: amenaza a un proyecto trascendente y ello puede desencadenar sacrificios y esfuerzo personales muy importantes. Los directivos no desaparecen sino que están más visibles y disponibles, particularmente antes los empleados y los clientes, para afrontar los desafíos.

De los relatos, también surge la evidencia que la superación de las crisis se asocia a cambios: no se vuelve a hacer lo mismo y puede constituir una oportunidad para nuevos desarrollos, como búsqueda de nuevos clientes (nacionales o extranjeros), fortalecer la relación con los trabajadores y clientes, apertura de nuevas líneas de negocio, etc.

\section{Construcción de red de relaciones (capital relacional)}

Conviene enfatizar que en situaciones de crisis generadas por entornos agresivos e incontrolables, la permanencia aparece asociada a la fortaleza de los lazos construidos por la organización con los stakeholders. Construir relaciones sanas y de confianza con las fuentes de crédito, los clientes, el personal y otros empresarios permite activar medidas para afrontar la crisis que de otra manera serían imposibles, especialmente cuando la crisis es del conjunto de la economía más que del ciclo de negocios del área de la empresa. Como se presenta más adelante, la relación con el estado también cuenta. Como ya se indicó, en la relación con el personal, los comportamientos testimoniales y ejemplares de la dirección adquieren fuerte relevancia.

\section{Relación con el estado / administración pública}

La presencia del estado aparece en tres aspectos: regulador de la actividad, cliente y árbitro en las relaciones laborales generando en consecuencia diferentes tipos de relaciones, pero sea en forma directa, sea a través de las asociaciones empresarias, los directivos mantienen fuertes 
contactos con la administración pública. Las empresas más reguladas parecen cambiar menos el negocio: los procesos de mejora están más en el primer nivel (mejoras de procesos y gestión) que en nuevos (modelos de) negocios. Incluso, la regulación puede aparecer como una suerte de garantía en situaciones de crisis, pues la regulación suele implicar un compromiso de la administración pública con el regulado. El estado como cliente tiene ventajas e inconvenientes: la escala, las regulaciones, los pagos, etc. que pueden ofrecer amenazas u oportunidades.

Ante esto, hay empresas que apuestan a no tener al estado como cliente por el grado de incertidumbre que puede provocar. En cuanto a las relaciones laborales, el conocimiento de los mecanismos y dinámicas de la administración pública aparece como clave.

\section{Discusión}

6.1 Propuesta para la clasificación de las migraciones de negocios De los relatos empresariales, surge que los estilos y prácticas de dirección encontrados tienen que ser interpretados en el marco de una dinámica de migración empresarial, entendidos como sustitución de negocios y/o modificación de los modelos de negocios. Las empresas entrevistadas han podido subsistir gracias a profundas transformaciones, siendo a veces más fieles a una voluntad de permanencia que a un negocio en particular y los perfiles de dirección relevados deben interpretarse desde procesos de gestión de cambios profundos en las organizaciones.

Los casos estudiados permiten construir 4 tipos de relación empresanegocio, que una misma organización puede haber aplicado en forma combinada o en diferentes momentos de su vida.

\section{Caso 1: Mejora de la gestión modelo de negocio}

La organización permanece largos períodos de tiempo con el mismo modelo de negocio, incluyendo ajustes y mejoras al paso del tiempo. La clave de su permanencia radica en la articulación entre un entorno relativamente estable, alta regulación pública y mantenimiento de posiciones dominantes. La organización no tiene presiones para cambiar su modelo de negocio. Si la empresa es grande, una de las claves de la sustentabilidad se encuentra en la capacidad de generar economías de escalas. Otro de los elementos clave, radica en la capacidad de influir en las reglamentaciones que afecten su actividad. La primera es más importante si el entorno es competitivo, mientras que la segunda es necesaria si las reglamentaciones además de regular la actividad, determinan márgenes de ganancia y condiciones de permanencia.

\section{Caso 2: Cambio del modelo de negocio}

El cambio del modelo de negocio está asociado múltiples factores: respuesta a nuevos entrantes, cambios tecnológicos, etc. La empresa sigue ofreciendo básicamente los mismos servicios o productos al cliente, pero modifica el modelo de negocio, ofreciendo nuevos canales, renovando productos, etc. Comparte aspectos del caso 1, como puede ser crecimiento de escala, mejora de procesos, pero implica cambios más fuertes.

\section{Caso 3: Extensión del (modelo) de negocio}

La ampliación del (modelo de) negocio se puede realizar en 3 direcciones: vertical, horizontal y geográfica. La ampliación vertical comprende la incorporación de actividad asociadas a la cadena de valor, tanto hacia abajo (suministro de insumos) como hacia arriba (procesamiento de productos, ampliación de la distribución, acceso al consumidor final, etc.). La ampliación horizontal comprende el proceso de incorporar nuevos negocios que no se asocian a la cadena de valor de otro negocio ya implementado, pero al cual el negocio madre ofrece apoyos, como acceso a clientes, etc. La tercer modalidad de extensión del negocio sucede cuando la empresa es capaz de instalarse en otras áreas geográficas, particularmente en el extranjero. Esta última es más relevante, ya que implica mayores desafíos, riesgos y a veces ello es posible siempre y cuando no se copie el modelo de negocio original.

\section{Caso 4: La migración organizacional}

En este caso la organización abandona su/s negocio/s clave e inicia otro negocio. Puede parecerse al caso 3, pero la diferencia fundamental es que el negocio original permanece marginal o desaparece: el corazón de la organización palpita en otro negocio. Aparece en forma pura cuando la organización muda a otro negocio, pero también existe en formas mitigadas cuando un grupo de la organización madre la abandona para construir otro emprendimiento. En su caso más fuerte y puro, la organización asume otro negocio orientándose a sectores con mayores oportunidades del momento y/o del ciclo de negocios (Smit et al., 2005).

\subsection{Propuestas para la gestión}

Las empresas entrevistadas, en general son empresas familiares que responden a un segmento caracterizado por la permanencia al tiempo y que proveen un razonable nivel de satisfacción al conjunto de los grupos de interés.

La sustentabilidad de este tipo de empresas y de directivos/empresarios es muy relevante para el conjunto de la sociedad. Permiten la existencia y fortalecimiento de un empresariado nacional, que provisto de competencias de gestión adecuadas es un actor relevante del desarrollo económico y social. Su ausencia significaría que entre las empresas del estado y las filiales de las empresas multinacionales y globales quedaría un vacío. No es evidente que una economía polarizada entre empresas públicas y multinacionales extranjeras tenga la dinámica económica requerida ni brinde oportunidades suficientes a las imprescindibles personas de perfil emprendedor.

En el contexto actual de la sociedad uruguaya, implica el fortalecimiento de una cultura de gestión organizacional que entre otros rasgos, convienen destacar:

1) Ser líderes de personas: las competencias técnicas o la habilidad comercial (aunque necesarias) no son suficientes para construir este tipo organización. El "alineamiento" de la organización fracasa sin la capacidad de motivar. Pero la motivación no puede 
ser ingenua: hay que reconocer la existencia de conflicto de intereses entre los propietarios y los trabajadores. El desafío es cómo se gestiona dicho conflicto ya que no es plenamente superable (Adizes, 1994). Esto no implica la imposibilidad de construir relaciones de ganar-ganar, pero sólo es posible hacerlo de manera realista. El compromiso altamente personal del empresario asociado a este tipo de empresa, ¿hasta dónde puede ser asumido por las otras personas?

Otra desafío importante que tiene que asumir este liderazgo dice a las diferencias generacionales a través de la creación no sólo de procesos de socialización laboral apropiados, sino también reconocer que la organización debe cambiar para adaptarse a características de las nuevas generaciones.

Estamos en un ciclo de negocios en el cual la gestión de personas es un aspecto que insume cada vez más tiempo, energía, recursos y competencias de cualquier directivo.

2) Coherente con el punto anterior, hay que aceptar que se está en presencia de un desafío para las relaciones laborales, que requieren superar dinámicas suma- cero por la construcción de dinámicas ganar-ganar en un compromiso con la organización de la cual directivos, trabajadores y sindicatos son responsables, cada uno en su campo. El estado tiene un papel imprescindible para fortalecer relaciones constructivas.

El ejemplo de la dificultad que indican los directivos para abordar los casos de bajo rendimiento, muchas veces aparecen asociados a las resistencias sindicales. Pero, ¿ ¿realmente ganan los trabajadores productivos y responsables conviviendo con los otros? Algunos ejemplos de alto rendimiento organizacional vienen acompañados de relatos en las cuales los propios trabajadores resisten la presencia de compañeros de bajo rendimiento o débil compromiso.

También son significativas las dificultades para manejar y difundir en el ámbito organizacional la información de empresa. Es un círculo vicioso, no comunicar genera desconfianza que fortalece la incomunicación. Pero el círculo virtuoso no necesariamente arranca por el mero hecho de comunicar.

3) Es común indicar que el cambio es permanente, pero igual hay que destacar este aspecto y en lo que concierne este estudio, aparece interesante la separación entre organización y negocio, procurando que la empresa adquiera competencias que le permitan al límite, migrar de negocio. En una perspectiva de responsabilidad social esta competencia es clave. Es la que permite que los costos en las personas, en sus familias y en la comunidad en la cual se alojan las empresas se minimicen cuando las mismas deban afrontar transformaciones críticas. La identificación del proyecto personal ( $\mathrm{y}$ a veces familiar trans-generacional) como una de las claves de la permanencia, vuelve a destacar la necesidad de un empresariado nacional con un perfil propio.
4) El cambio permanente observado en los modelos de negocios confirma la necesidad de considerar a los mismos como herramientas de la empresa más que la identidad de la empresa. La gestión del modelo de negocios resulta una herramienta a incorporar en la estrategia organizativa.

5) Las empresas observadas tienden a seguir un crecimiento orgánico (Äijö et al., 2005), es decir basado en recursos propios o con financiamiento externo que no afecta la propiedad. Esta práctica puede construir una especie de techo de cristal que limita el crecimiento y en particular para ciertas empresas, dificulta la posible o necesaria internacionalización. Pero la experiencia de empresas nacionales con internacionalización en forma radical, con la pérdida de la mayoría propietaria, muestra que los propietarios extranjeros (usualmente multinacionales) pueden llevar a rupturas de las culturas que generaron el éxito, a prácticas de reducción de la empresa, cuando no el cierre definitivo.

6) En una mirada a largo plazo las organizaciones deben saber que tendrán que afrontar crisis y la conciencia (no terrorista) de que tendrán momentos particularmente difíciles debe ser parte de la cultura de la organización. Más allá de los aspectos estrictamente financieros, se aprende de las empresas entrevistadas, que una de las claves para afrontar estas situaciones es la construcción de un fuerte capital relacional. La organización que genera confianza en el conjunto de sus grupos de interés cuenta con ventajas y recursos para afrontar las crisis. También se observa que cierto desapego al negocio o al modelo de negocio utilizado, puede generar oportunidades en situación de crisis y que el apego al mismo constituye una barrera: no es lo mismo ajustarse, para luego seguir haciendo lo mismo, que ajustarse para hacer cosas nuevas.

7) La sustentabilidad de las organizaciones no depende únicamente de las competencias de la dirección. El comportamiento del personal y en particular de sus organizaciones también impactan en la permanencia en el tiempo. El continuo estado de (inminencia de) conflicto constituye un lastre organizacional. No son raros los casos de empresas o filiales multinacionales que tienden a cerrar o reducir sus actividades por este tipo de situación. Pero muchos directivos destacan también la energía y el tiempo que lleva el nuevo estado de la gestión de los recursos humanos y como este esfuerzo tiene un costo de oportunidad para otro tipo de iniciativas y mejoras competitivas. Como ya se indicó, el estado debería cumplir un papel importante en la creación de relaciones laborales de nuevo tipo.

\subsection{Propuestas para las escuelas de negocios}

Esta investigación muestra que la sustentabilidad organizacional observada en el Uruguay puede tener perfiles propios con mayor o menor ajuste a las tendencias observadas en los países dominantes y en la literatura de las grandes empresas multinacionales. Si esto es una manifestación más de las especificidades propias de cada nación, se sigue que las escuelas de negocios deben ser capaces de captar la especificidad nacional generando y trasmitiendo un conocimiento situado propio que no implica estar cerrado al pensamiento global. 
La sustentabilidad organizacional requiere competencias específicas que deben ser tratadas no sólo en términos de investigación, sino también de docencia y aprendizaje. La creciente profesionalización de la gestión empresarial, manifestada tanto por el auge de las escuelas de negocios como por las formas de reclutamiento del personal superior y en el marco de la investigación, el perfil crecientemente profesional de las sucesivas generaciones que ocupan los cargos de dirección de las empresas, confirman el papel protagónico de la educación.

Entre las características específicas que importa trabajar e investigar en estas escuelas se puede destacar para el Uruguay, que a veces las teorías de la gestión y los manuales más generalizados en medios académicos, parecen tener dificultades para reconocer que las organizaciones empresariales son organizaciones conflictivas, en las cuales hay enfrentamientos de interés entre las diferentes partes, accionistas, directivos y trabajadores. Negar estos conflictos de interés pueden transformar las ciencias empresariales en una ideología. Superar el enfoque ideológico constituye un aspecto clave para abordar la sustentabilidad. Los estudiantes deben ser conscientes de estos conflictos y adquirir competencias para abordarlos, no sólo técnicas sino éticas.

Una vez más, se confirma que la formación empresarial requiere una dimensión de liderazgo, tanto en sus aspectos prácticos como en las habilidades personales y su articulación con los valores. No existen comportamientos o prácticas que impliquen personas que no tengan una dimensión ética.

\subsection{Limitaciones y futuras investigaciones}

Si bien desde el armado del marco teórico se es consciente que el modelo utilizado basado en competencias de gestión no es exhaustivo -es decir que la sustentabilidad depende también de otros factores no incluidos- importa destacar que desde el material generado por el estudio queda en evidencia que las condiciones de entorno general o específico de la organización y las capacidades operativas son clave. En estas últimas se puede destacar la habilidad de la dirección de identificar oportunidades de negocio y actuar rápidamente en consecuencia. La importancia de la gestión del modelo de negocio es un aspecto de ello, pues no basta con tener un modelo apropiado, sino que también hay que saber gestionarlo.

La investigación tiene básicamente un diseño exploratorio y descriptivo. Una manera de avanzar es: fortalecer la modelización; generar hipótesis; expandir el estudio a otro tipo de empresas. La tipología de migraciones de modelos de negocios en el Uruguay constituye una primera aproximación que amerita mayor profundización, especialmente por el potencial de uso que tiene la herramienta de la construcción y gestión modelos de negocios.

El estudio constató problemas asociados a las limitaciones del crecimiento orgánico y dificultades cuando no resistencias para aprovechar las oportunidades de internacionalización desde el Uruguay hacia el exterior. Esto constituye una debilidad de parte del empresariado nacional y se asocia a los problemas que tienen los pequeños países emergentes para que sus empresas crezcan más allá de sus fronteras. No resolver estas limitaciones constituye una amenaza a las condiciones de existencia y desarrollo de un empresariado nacional.

\section{Agradecimientos}

El equipo investigador y la Universidad Católica del Uruguay agradecen muy especialmente a todos los empresarios entrevistados quienes se esmeran día a día por alcanzar estándares de desempeño superior en sus organizaciones, siempre dispuestos a aprender y compartir sus experiencias con la academia y con otros empresarios, su comportamiento resulta ejemplificador en el camino de las buenas prácticas económicamente sustentable y socialmente responsable.

El equipo investigador también agradece a la Universidad Católica del Uruguay por el aval institucional brindado a la línea de investigación Organizaciones de Alto Desempeño, del Departamento Ciencias de la Administración, de la Facultad de Ciencias Empresarial, permitiendo la continua producción y difusión de conocimiento en gestión sustentable y dirección empresarial responsable desde el año 2003.

\section{Bibliografía}

Adizes, I. (1994). Ciclos de vida de la organización: cómo y por qué crecen y mueren las organizaciones y qué hacer al respecto. Madrid: Díaz de Santos.

Äijö, T., Kuivalainen, O., Saarenketo, S., Lindqvist, J., \& Hanninen, H. (2005). Internationalization Handbook for the Software Business. Espoo, Finland: CESPB.

Algorta, M. et al. (2012). Prácticas de gestión que dan resultados. La experiencia de organizaciones de alto desempeño en el Uruguay. Montevideo: UCU/Ed. Grupo Magro.

Barton, D., \& Wiseman, M. (2014). Focusing capital on the long term. Harvard Business Review, 92(1/2), 44-51.

Brady, H. E. Y Collier, D. (2010). Rethinking social inquiry. Diverse Tools, Shared Standards. Plymouth, UK: Rowman and Littlefield Pub. (2nd ed.)

Brynjolfsson, E., y McAfee, A. (2014). The Second Machine Age, NY:W.W. Norton\&Company.

Carayannis, E.G., Sindakis, S., Y Walter, C. (2014). Business Model Innovation as Lever of Organizational Sustainability. Journal of Technology Transfer. DOI 10.1007/s10961-013-9330-y, published on-line.

Davis, I. (2014a). Reflections on corporate longevity. McKinsey Quaterly, (3) 118-122.

Davis, I. (2014b). Lou Gerstner on corporate reinvention and values. McKinsey Quaterly, (3) 123-129. 
De Waal. A. 2012. What Makes a High Performance Organization: Five Factors of Competitive Advantage That Apply Worldwide. Hilversum: Financial World Publishing.

Durán, C.; Jung, A. y García, S. (2012) Igualdad de oportunidades y construcción de capacidades para el desarrollo. El caso de Uruguay. En: Pobreza y desigualdad de oportunidades y políticas públicas en América Latina. Rio de Janeiro:Konrad Adenauer Stiftung. pp 285-204

Gunther McGrath, R. (2013). The End of Competitive Advantage: How to Keep Your Strategy Moving as Fast as Your Business. New York: Harvard Business Review Press.

Hesselbein, F., Goldsmith, M., y Beckhard, R. (1998). La organización del futuro. Buenos Aires: Fundación Drucker, Granica.

Man, T.W. y Lau, T. (2000). Entrepreneurial competencies of SME owner/manager in the Hong Kong services sector: a qualitative analysis. Journal of Enterprising Culture. 8 (3) 235-254.

Miles J. (2011). Análisis del Capital Intelectual de las Pequeñas y Medianas Empresas Uruguayas y su Impacto en los Resultados. Un estudio en las empresas desarrolladoras de software. Tesis Doctoral. Universidad de Deusto.

Mintzberg, H. (1983). Power in and around organizations. Englewood Cliffs, N. J.: Prentice-Hall.

Osterwalder, A., y Pigneur, Y. (2010). Generación de modelos de negocio. Barcelona: Centro Libros PAPF.
Rosenbusch, N., Bausch, A. y Galander, A. (2007). The impact of environmental characteristics on firm performance: a meta-analysis of empirical findings. Paper presented at Academy of Management Annual Meeting 2008, Anaheim, United States.

Shafer, S. M., Smith, H. J., \& Linder, J. C. (2005). The power of business models. Business Horizons, 48(3), 199-207.

Smit, S., Thompson, C., y Viguerie, P. (2005). The do-or-die struggle for growth. McKinsey Quaterly, (3), 33-45.

Teece, D. \& Pisano, G. (1994). The Dynamic Capabilities of Firms: An Introduction. Industrial and Corporate Change, (3) 3, 537-556.

Helfat, C., Finkelstein, S., Mitchell, W., Peteraf, M., Singh, H., Teece, D., Winter, S. (2007). Dynamic capabilities: Understanding Strategic Change In Organizations. Oxford: Blackwell Publishing.

Teece, D. (2009). Dynamic capabilities and strategic management. Oxford: Oxford University Press.

Thomas, T., \& Lamm, E. (2012). Legitimacy and Organizational Sustainability. Journal of Business Ethics (2012) 110:191-203.

Vázquez, S. (2015). El caso de Uruguay. En: Política monetaria y fiscal. ¿Ha aprendido América Latina a mitigar los efectos de las crisis? Sopla/ KAS. (En edición).

Wikström, P. A. (2010). Sustainability and Organizational Activities Three Approaches. Sustainable Development, 18, 99-107. 